\title{
Unusual case of ruptured sinus of valsalva involving left and right sinus of valsalva in to left ventricle
}

\begin{abstract}
Sinus of Valsalva aneurysm is a rare congenital disease. Rupture of right sinus of valsalva is the most common type and rupture of left sinus of valsalva is the rarest type. We report an extremely rare case involving right and left coronary sinuses of valsalva into left ventricle with moderate aortic regurgitation in a 21 year old male. $\mathrm{He}$ underwent successful autologous pericardial patch repair and closure of ruptured aneurysm with concomitant aortic valve replacement. The rarity of this case highlights the requirement for a precise preoperative diagnosis, the role of echocardiography and last but not least the importance of prompt surgical management
\end{abstract}

Keywords: rupture sinus of valsalva, aortic regurgitation, congenital heart disease, left sinus of valsalva, right sinus of valsalva, hypokinesia, moderate aortic regurgitation, intraoperative tee, aorto-mitral curtain, pledgetted polypropyle suture, non-coronary sinus

\author{
Volume 6 Issue 5 - 2018

\begin{abstract}
Nitin Gupta,' Vijaykumar Raju,' Koushik Kannnan,' Kalyanasundaram Muthuswamy, Muralidharan Srinivasan'

'Department of Cardiothoracic Surgery, G. Kuppusamy Naidu Memorial Hospital, India

${ }^{2}$ Department of Paediatric Cardiology, G. Kuppusamy Naidu Memorial Hospital, India
\end{abstract}

\author{
Correspondence: Vijayakumar Raju, Consultant \\ Cardiothoracic Surgeon, GKNM Hospital, Coimbatore, Tamil \\ Nadu, India, 64I037, Tel +9445I06484, \\ Email vizraju@Hotmail.com
}

Received: February 25, 2018 | Published: November 16, 2018
Abbreviations: MAR, moderate aortic regurgitation; NYHA, new york heart association; TEE, trans esophageal echocardiography; $\mathrm{LV}$, left ventricle; AMC, aorto-mitral curtain; CHD, congenital heart disease

\section{Case report}

A 21 year old male was evaluated for Dyspnoea on Exertion NYHA class II since 3 months duration which progressed to class III for the past 2 weeks. On examination, he was thin built with height of $179 \mathrm{~cm}$. His blood pressure was $160 / 40 \mathrm{mmHg}$. There were no other features suggestive of Marfan Syndrome or any other connective tissue disorder. There was thrill present in the left 3 intercostal space just left of the sternum. Early diastolic murmur was well heard in the same area. Chest X-ray showed cardiomegaly. Echocardiogram revealed rupture of Left Sinus of Valsalva into the Left Ventricle, Moderate Aortic Regurgitation with global hypokinesia of left ventricle and severe Pulmonary artery hypertension. He was taken up for surgical repair of rupture sinus of valsalva and possible aortic valve repair. Intraoperative TEE showed moderate to severe Aortic regurgitation with no other intracardiac lesion but it was difficult to determine whether this was single or multiple ruptured sinus of Valsalva. Through midline sternotomy and after harvesting pericardium, Cardiopulmonary bypass was established with aortic and bicaval anastomosis. LV vent was placed through right superior pulmonary vein. The intraoperative examination through a transverse aortotomy revealed thickened and retracted tricuspid aortic valve with dilated aortic annulus with lack of central coaptation. There was about $20 \times 15 \mathrm{~mm}$ defect in the left sinus of valsalva with about $2 \mathrm{~cm}$ length of wind-sock into left ventricle. The wind-sock was looking unhealthy and rupturing into the Left ventricle. The windsock was occupying the entire region of Aorto-Mitral Curtain. About $5 \times 3 \mathrm{~mm}$ size defect was noted in the right sinus of Valsalva with dilated aortic annulus. Both the wind-sock was identified into the Left ventricle and its rupture was confirmed. Through aortic root the entire windsock from the left sinus was excised and it was closed directly using polypropylene sutures. The defect in the left sinus of valsalva was closed using appropriate size pericardial patch with Polypropylene suture. The additional rupture of sinus of valsalva in the right coronary sinus was closed directly using pledgetted polypropyle suture. On saline test we found that there mild central aortic regurgitation. We came off cardiopulmonary bypass after routine de-airing of the left side of the heart. TEE showed no residual shunt across the repaired sinus but there was persisting more than moderate aortic regurgitation with lack of central coaptation. In view of significant residual aortic regurgitation, we went back on Cardiopulmonary bypass and replaced the aortic valve with mechanical valve. The post-operative course was uneventful and the patient was discharged in healthy condition on the sixth post operative day. Pre-discharge Echocardiogram showed with no residual ruptured sinus of valsalva and well functioning prosthetic valve. On three months follow up, he is doing well and remains in NYHA class I (Figure 1).

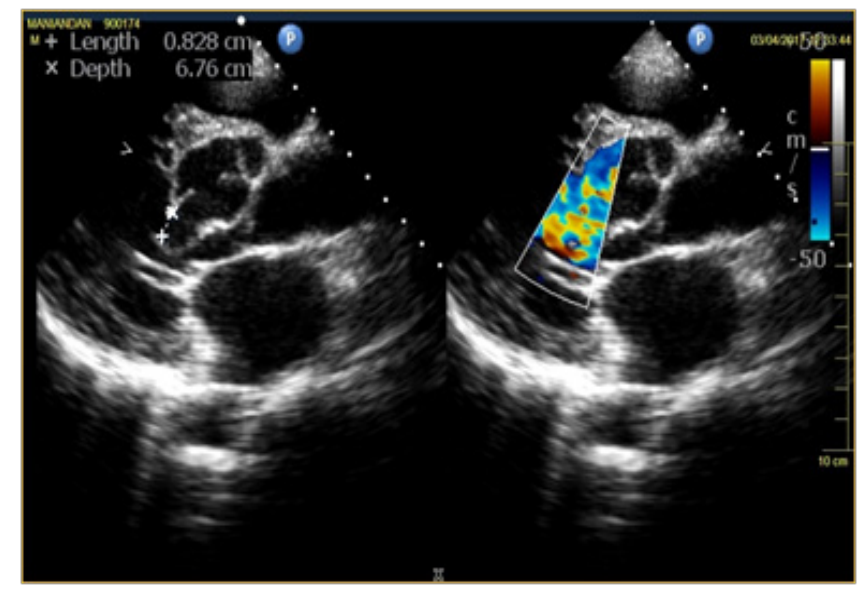

Figure I 2-D Echo with colour Doppler showing rupture of sinus valsalva from the left sinus.

\section{Discussion}

Sinus of Valsalva aneurysm is a rare congenital anomaly presents approximately in $0.09 \%$ of population which occurs due to separation between the aortic media and the annulus ${ }^{1}$. It can be a silent asymptomatic aneurysm and detected incidentally or present as a catastrophic rupture in heart failure. The right sinus of Valsalva is most 
commonly involved $(76.8 \%)$, followed by the non-coronary sinus, and, least commonly, the left coronary sinus $(3 \%)^{2}$. Simultaneous involvement of right sinus and left sinus with rupture in to the left ventricle has not been reported in literature as far as our knowledge goes. Ruptured sinus of Valsalva aneurysm is rare but is usually diagnosed on echocardiography. Sinus of Valsalva has a higher male preponderance (4:1) and the incidence is higher in Asian populations ${ }^{2}$. The etiologycan be either congenital or acquired. Acquired conditions occurs less frequently and is usually caused by diseases affecting the aortic wall, such as infection (syphilis, bacterial or fungal endocarditis, and tuberculosis), degenerative disease (atherosclerosis, connective tissue disorders, and cystic medial necrosis), or thoracic trauma ${ }^{3,4}$. Unruptured conditions usually remain asymptomatic (Figure 2).
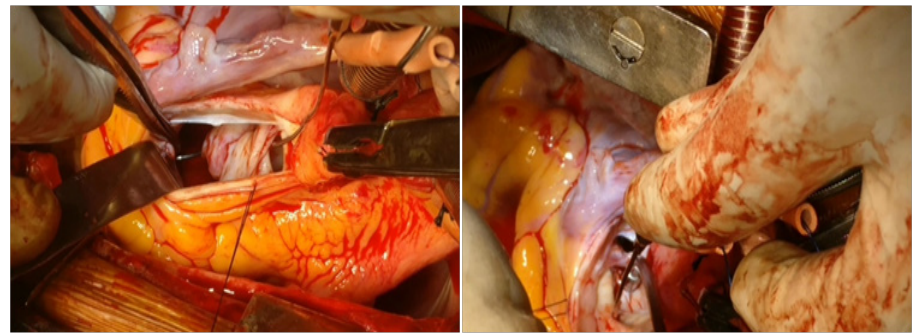

Figure 2 2A: Peroperative Probe demonstrating rupture of left sinus of valsalva in to the left ventricle.

$2 \mathrm{~B}$ : Peroperative probe demonstrating rupture of right sinus of valsalva in to the left ventricle.

In 1962, Sakakibara and Konno ${ }^{5}$ proposed the first formal classification system for sinus of Valsalva aneurysms, according to their site of origin and rupture. This anatomic classification system described only 4 types of aneurysms, arising from either the right coronary sinus or the non-coronary sinus, and did not include left sinus nor account for all possible chambers of penetration. The modified Sakakibara classification proposed by Xin-jin ${ }^{5}$ included Type 5 group which included other rare conditions (e.g., rupture into left atrium, pulmonary artery, left ventricle, or other structures). In the present case we found an additional sinus of Valsalva from right coronary sinus opening into left ventricle. We could not find any literature mentioning this condition to fit into any of the types even in the modified Sakakibara and Konno classification (Figure 3).

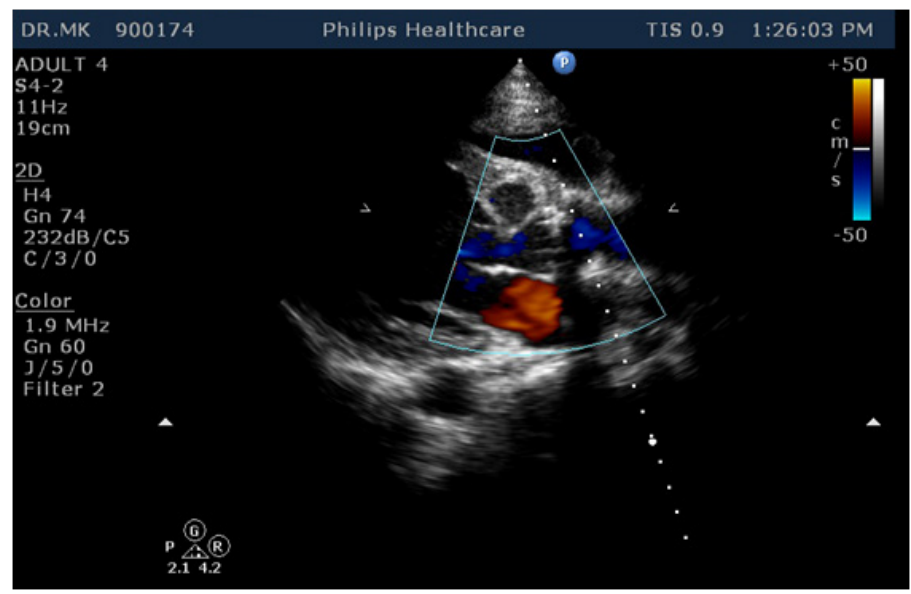

Figure 3 Postoperative Echo demonstrating no residual Rupture of sinus of valslava with well functioning mechanical valve in the aortic position.
As reported by Takach $^{6}$ about $40 \%$ of cases of rupture sinus of valsalva can have associated aortic regurgitation. The supra annular diastolic runoff is the usual cause of aortic regurgitation in the absence of other structural defect in the aortic valve. This aortic valve usually does not need any additional repair or the valve replacement. Patch closure of ruptured sinus alone will decrease the degree of aortic regurgitation. But our patient had thickened and retracted valve margin to start with probably with involvement of two sinus with rupture and did not have successful reduction in the degree aortic regurgitation even after closure of both ruptured sinus, justifies valve replacement.

\section{Conclusion}

Rupture of Sinus valsalva involving both left and right sinus of valsalva has not been reported so far in the literature. Involvement of more than one sinus with presence of thickened, retracted aortic valve leaflet with more than moderate aortic regurgitation mandates additional valve procedure in the form of repair or replacement along with repair of sinus of valsalva.

\section{Acknowledgements}

None.

\section{Conflict of interest}

Authors declare that there is no conflict of interest.

\section{References}

1. Feldman DN, Roman MJ. Aneurysms of the sinuses of Valsalva. Cardiology. 2006;106(2):73-81.

2. Chu SH, Hung CR, How SS, et al. Ruptured aneurysms of the sinus of Valsalva in oriental patients. J Thorac Cardiovasc Surg. 1990;99(2):288298.

3. C Batiste, R C Bansal, and A J Razzouk, "Echocardiographic features of an unruptured mycotic aneurysm of the right aortic sinus of Valsalva," .Journal of the American Society of Echocardiography. 2004;17(5):474477.

4. Greiss, P Ugolini, M Joyal, D et al. "Ruptured aneurysm of the left sinus of Valsalva discovered 41 years after a deceleration injury,". Journal of the American Society of Echocardiography. 2004:17(8):906-909.

5. Xin-Jin L, Xuan L, Bo P, et al. Modified Sakakibara classification system for ruptured sinus of Valsalva aneurysm. The Journal of Thorac Cardiovasc Surg. 2013:146(4):874-878.

6. Takach TJ, Reul GJ, Duncan JM, et al. Sinus of Valsalva aneurysm or fistula: management and outcome. Ann Thorac Surg. 1999;68(5):15731577 . 